\title{
Advances in Measuring Outcome for Children and Adolescents With Brain Injury
}

\author{
W. Huw Williams ${ }^{1,2}$ \\ ${ }^{1}$ School of Psychology, Washington Singer Labs, Exeter University, United Kingdom \\ ${ }^{2}$ Emergency Dept. Royal Devon and Exeter Hospital, United Kingdom
}

Clinicians, educators and researchers are very aware of the need for outcome measures to guide rehabilitative efforts for children and adolescents with brain injury. There are, of course, many challenges to such measurement. Outcome measures need to be reliable, sensitive and valid. That is, to measure what they purport to measure, and not other skills and abilities or behaviour, and to match onto the demands of everyday lives. Moreover, there is a need to take account of the changes one would expect with age, particularly in light of age at time of injury, time since injury and relevant processes and stages of neuro-development. There are, for example, critical periods for brain growth, potential for neuroplasticity and for crowding effects - that is, functions being compromised as neurological systems become utilised for alternate functions (see Anderson et al. [2009] and Tonks et al. [2009]). Socio-environmental resources are also important in understanding outcomes postacquired brain injury (ABI). Predicting developmental trajectories post-ABI is therefore complex, with a range of potential outcomes - from decline, plateau, reduced development through to resolution.

What is meant by outcome is also worth considering. 'Outcome' — particularly regarding the developing brain - is not a 'product' at which a child has reached, but is in transition through a 'process' (Williams, Evans \& Wilson, 1999). 'Outcome measures' provide guidance on how well they may have retained or re-developed skills so as to be able to maintain a role at home, school, or community. Importantly, measures provide a guide for knowing what skills are compromised and need to be compensated for. Such measures are also vital in that, at a broader level, those who plan and provide services can appropriately measure the range of issues faced by survivors of brain injury and their families.

This special issue of Brain Impairment provides important advances in the area of outcome measurement post-ABI for children and adolescents. Zivani et al. (2010) provide a systematic review of measures of participation and environmental outcomes for children with ABI. In their review, five measures of participation and six measures of environment were identified. Zivani et al. note how more work needs to be done to evaluate these measures so as to improve their clinical utility. Motor skills are crucial for everyday functioning - many children with neuro-disability may have difficulty in motor control and coordination. Davis et al. (2010) report a study on the reliability of the Assisting Hand Assessment (AHA) for children and youth with ABI. The AHA was developed with children who have Cerebral Palsy or Obstetric Brachial Plexus Palsy. It provides an evaluation of the function of a child or youth's assisting hand through observing the spontaneous manipulation of objects during bimanual play. In Davis et al.'s study it was found that the AHA had good interrater and intrarater reliability when used with the paediatric ABI population. This provides support for its continued use for children and youth with acquired hemiplegia.

Cognitive difficulties are, of course, the signature issue post-ABI. However, how to assess such skills in a way that both provides an index of everyday difficulty, and makes use of the observations of those in the child's environment, are not well developed. Chevignard et al. (2010) report a study on the development and evaluation of an ecological task to assess executive functioning the Children's Cooking Task (CCT). They note how sensitivity and ecological validity of neuropsychological tests of executive functions have

Address for correspondence: Associate Professor W. Huw Williams, School of Psychology, Washington Singer Labs, Exeter University, United Kingdom. E-mail: w.h.williams@ex.ac.uk 
been questioned and, therefore, there is a need for such ecological and open-ended assessments of executive functions. They report a matched control study within which children with moderate-tosevere traumatic brain injury (TBI), as well as children with mild TBI, made more errors compared to controls. Performance correlated with established executive measures. In the area of memory functions, Levick (2010) provides a review of observer rating of memory in children. He notes that there are critical psychometric issues and challenges to such research. These challenges mean that, for now, observer rating scales for everyday memory problems lack sensitivity and reliability. It is noted that more research is needed so that measures could be evolved that have stronger association with objective memory measures.

The area of social functioning is, of course, a critical area for any survivor of ABI. To be in a social role is the key end goal of neurorehabilitation and provides inherent reinforcement. There are many factors - cognitive, social, behavioural and environmental - that can conspire to limit the resocialisation of survivors. Crucially, socially appropriate and competent behaviour is required to maintain social roles. Dooley et al. (2010) describe a study on the measurement of sociomoral reasoning in adolescents with TBI. They note, rightly, how moral reasoning skills are important for appropriate and adaptive social functioning and that impairments in moral reasoning are linked to aggressive and violent behaviours. Dooley et al. describe new measures of sociomoral reasoning and maturity (SoMoral and So-Mature). Two studies are reported the first with a normative group, and second with TBI sample - which provide preliminary support for the validity and reliability of both tasks and preliminary evidence of utility with TBI groups. Social competence is addressed by Muscara et al. (2010). They note how social development is often affected by ABI but that there remains a lack of specific, relevant and robust measurement tools for such skills. They report a project designed to develop a questionnaire to measure social competence and the quality of relationships of children and adolescents, between the ages of 5 and 18 years, using parental reports. Preliminary analyses indicate that the measure shows promise. Specific communication problems would inevitably lead to difficulties in engagement and socialisation - and impact on education and so on. Douglas (2010) describes the La Trobe Communication Questionnaire which was designed to measure perceived social communication abilities from various sources - including selfperceptions as well as perceptions of others (e.g., family members, friends and clinicians). In this study it was shown that the measure was sensitive in measurement of differences between adolescents with TBI and noninjured, suggesting that it may be a valuable tool for determining social communication difficulties post- TBI.

An area of vital importance is that of care and support needs of children with ABI. Only through the use of such measures can the real costs and benefits of neurorehabilitative efforts be quantified such that service providers and purchasers can meet the needs of survivors and their families. Soo et al. (2010) describe the Paediatric Care and Needs Scale (PCANS), which was developed to address the lack of scales available for measuring support needs. The scale assesses supervision and physical assistance across 14 domains of everyday activities - and addresses, for example, support for personal hygiene through to participation in leisure and social activities. Soo et al. (2010) describe a study that provides normative data for the PCANS using a sample of children stratified by age.

This special issue of Brain Impairment therefore provides a valuable resource of measures that can be used to monitor and guide neurorehabilitative efforts with children and adolescents with brain injury. The use of such measures can thus contribute to enabling those injured to be provided with appropriate resources and support so as gain the skills and opportunities to optimise their outcome - to have a place in society.

\section{References}

Anderson, V., Spencer-Smith, M., Leventer, R., Coleman, L., Anderson, P., Williams, J., Greenham, M., \& Jacobs, R. (2009) Childhood brain insult: Can age at insult help us predict outcome? Brain, 132, 1, 45-56.

Tonks. J., Slater, A., Frampton, I., Wall, S., Yates, P., \& Williams, W.H. (2009). The development of emotion and empathy skills after childhood brain injury. Developmental Medicine \& Child Neurology, 51 (1), 8-16.

Williams, W.H., Evans, J.J. \& Wilson, B.A. (1999). Outcome measures for survivors of acquired brain injury in day and outpatient neurorehabilitation programmes. Neuropsychological Rehabilitation, 9, 421-436. 